\title{
Localization of dexamethasone within dendritic core-multishell (CMS) nanoparticles and skin penetration properties studied by multi-frequency electron paramagnetic resonance spectroscopy (EPR)
}

\author{
S. Saeidpour ${ }^{\mathrm{a}}$, S.B. Lohan ${ }^{\mathrm{b}}$, M. Anske ${ }^{\mathrm{a}, \mathrm{b}}$, M. Unbehauen ${ }^{\mathrm{c}}$, E. Fleige $^{\mathrm{c}}, \mathrm{R}$. \\ Haag $^{\mathrm{c}}$, M.C. Meinke ${ }^{\mathrm{b}}$, R. Bittl ${ }^{\mathrm{a}}$, C. Teutloff ${ }^{\mathrm{a}}$ \\ ${ }^{a}$ Berlin Joint EPR Lab, Freie Universität Berlin, Fachbereich Physik, Berlin, Germany \\ ${ }^{b}$ Charité - Universitätsmedizin Berlin, Department of Dermatology, Venerology and \\ Allergology, Center of Experimental and Applied Cutaneous Physiology, Berlin, Germany \\ ${ }^{c}$ Freie Universität Berlin, Institut für Chemie und Biochemie, Berlin, Germany
}

\begin{abstract}
The skin and especially the stratum corneum (SC) act as a barrier and protect epidermal cells and thus the whole body against xenobiotica of the external environment. Topical skin treatment requires an efficient drug delivery system (DDS). Polymer-based nanocarriers represent novel transport vehicles for dermal application of drugs. In this study dendritic core-multishell (CMS) nanoparticles were investigated as promising candidates. CMS were loaded with a drug (analogue) and were applied to penetration studies of skin. We determined by dual-frequency electron paramagnetic resonance (EPR) how Dexamethasone (Dx) labelled with 3-carboxy-2,2,5,5-tetramethyl-1-pyrrolidinyloxy (PCA) is associated with the CMS. The micro-environment of the drug loaded to CMS nanoparticles was investigated by pulsed high-field EPR at cryogenic temperature, making use of the fact that magnetic parameters ( $g$-, $A$-matrices, and spin-lattice relaxation time) represent specific probes for
\end{abstract}


the micro-environment. Additionally, the rotational correlation time of spinlabelled Dx was probed by continuous wave EPR at ambient temperature, which provides independent information on the drug environment. Furthermore, the penetration depth of Dx into the stratum corneum of porcine skin after different topical applications was investigated. The location of Dx in the CMS nanoparticles is revealed and the function of CMS as penetration enhancers for topical application is shown.

\section{Keywords:}

Drug delivery system (DDS), dexamethasone, dendritic core-multishell systems (CMS), dual-frequency EPR, skin penetration, solvent polarity, mobility

\section{Introduction}

Today delivery and controlled release of drugs to specific targets are im3 portant fields in medical, pharmaceutical, and multi-disciplinary research. 4 Different carrier systems have been developed in the last decades, ranging 5 from macromolecules to nanoparticles. These vehicles can improve the up6 take and penetration of drugs into the skin and reduce side effects [1-3] 7 Especially for the hair follicles, an improved penetration could be demon8 strated [4-7] Pharmaceutically used drugs show various physical and chemi9 cal properties, thus the use of carrier systems is depending on the respective drug and its application [8]. Polymer-based nanocarriers are candidates for drug delivery systems (DDS) [9-11] and have various advantages: drug carrier development is less time and cost demanding compared to new and more 
efficient drugs; nanocarriers can reduce drug side effects and can enhance treatment efficiency of diseases due to lower dose requirements. Another nanocarrier advantage is the protection of drugs against degradation before release at the target [12]. Furthermore, they enable the transport of a multitude of different drugs with different chemical properties [13].

Dendritic core-multishell (CMS) nanoparticles represent a class of new nanoparticles applicable as DDS $[9,14]$. These particles consist of a dendritic polyglycerol core with polar properties, which is surrounded by two layers of different chemical composition, allowing the transport and storage of molecules with lipophilic (inner shell) or hydrophilic (outer shell) character [15-17]. Recently, these CMS nanoparticles have successfully been used for dermal application. Küchler et al. could show an increased penetration for the lipophilic fluorescence dye nile red loaded to CMS particles into porcine skin compared to a base cream or solid lipid nanoparticles (SLN) [18]. Additionally, an increased penetration for 3-carboxy-2,2,5,5-tetramethyl-1-pyrrolidinyloxy (PCA) loaded to CMS nanoparticles was shown in comparison to ultra flexible vesicles and aqueous solution by EPR [14].

Two major questions have to be addressed for future applications of CMS as DDS in topical treatments: 1 . How can a pharmaceutical be efficiently loaded to the DDS, and 2. how is it released upon application onto the target? A prerequisite for answering both questions is knowledge about the location of the drug within the DDS and its mobility before and after topical application.

Electron paramagnetic resonance (EPR) spectroscopy is a well-developed spectroscopic method for the determination of the micro-environment of a 
paramagnetic molecule [19, 20].

The applicability of spin labels as probes within a carrier system and the analysis of their penetration profile into excised porcine ear skin was previously shown $[14,17,21]$. In the present study the location of pharmaceuticals in CMS nanoparticles, the drug penetration into the porcine ear skin, and the possible release of the drug to the strateum corneum (SC) were investigated by dual-frequency EPR spectroscopy. As a model drug we used the lipophilic $(\log \mathrm{P}=1.83)$ [22] antiinflammatory glucocorticoid Dexamethasone $(\mathrm{Dx})$. Since $\mathrm{Dx}$ is diamagnetic and thus not EPR active it was labelled by the spin marker PCA, which increases the molecular weight form 392.47 $\mathrm{g} / \mathrm{mol}$ for Dx to $560.68 \mathrm{~g} / \mathrm{mol}$ for DxPCA. Even tough PCA is a hydrophilic compound $(\log \mathrm{P}=-1.7[14,23])$, its attachment to Dx by esterification [24] leaves the partitioning coefficient almost unaltered $(\log \mathrm{P}=1.89 \pm 0.02$ for DxPCA [25]). The Zeeman interaction and the prominent nitrogen hyperfine coupling ( $g$ - and $A$-matrix) as well as the spin-lattice relaxation time of the unpaired electron on a nitroxide are highly sensitive probes for the surrounding micro-environment properties. [26, 27]. We investigated these magnetic parameters for DxPCA by pulsed W-band (94 GHz) EPR spectroscopy at cryogenic temperature $(80 \mathrm{~K})$. Room temperature EPR spectra at X-band $[28,29]$ were used to investigate the drug mobility within the carrier and its penetration into the porcine ear skin. The latter is possible since the rate of $\mathrm{PCA}$ reaction with the skin antioxidant system is slow enough to allow investigations on porcine ear skin [30, 31]. 


\section{Materials and Methods}

\subsection{Sample preparation}

The synthesis of DxPCA will be described elsewhere [24]. DxPCA was loaded to CMS nanoparticles by an entrapment film method: First, DxPCA was dissolved in ethanol, afterwards the solvent was evaporated and dried under vacuum condition yielding a thin DxPCA film on the surface of the glass container. Second, the CMS nanoparticles in aqueous solution $(5 \mathrm{~g} / \mathrm{L})$ were added to the DxPCA thin film. The solution was stirred for 22 hours at $1200 \mathrm{rpm}$. At the end, the solution was filtered through a $0.45 \mu \mathrm{m}$ regenerated cellulose $(\mathrm{RC})$ filter.

The concentration of DxPCA in different solvents used as reference samples was $50 \mu \mathrm{M}$, except for the solvent toluene where it was $25 \mu \mathrm{M}$. DxPCA dissolves only poorly in water (already Dx has a poor solubility of $89 \mathrm{mg} / \mathrm{L}$ at room temperature [32]). Thus, for achieving the desired DxPCA concentration in water, the solution was sonicated and heated up to $70{ }^{\circ} \mathrm{C}$ for thirty minutes and afterwards diluted. Finally, this solution was centrifuged and aggregated parts were removed by taking the supernatant.

The samples used for the measurements at W-band frequencies were frozen immediately before the experiments in liquid nitrogen. For improving the sample homogeneity upon freezing, glycerol was added in a 1:1 ratio to the aqueous DxPCA and PCA solution. All solvents (purity $\geq 98 \%$ ) used were purchased from Sigma-Aldrich. 


\subsection{Skin preparation}

For the skin penetration studies porcine ear skin was utilised, which was shown to be a suitable model for human skin [33, 34]. The porcine ears were delivered by a local butcher with approval by the Veterinäramt Berlin (Treptow-Köpenick) on the day of slaughter and were cleaned as previously described [17]. For the EPR measurements only the backside of the ears was used. The uppermost skin layer of the porcine ears was cut-off by a dermatome (Dermatom Typ GA 140, Aesculap-Werke AG, Tuttlingen, Germany) in a thickness of around $400 \mu \mathrm{m}$. For the penetration studies, $90 \mu \mathrm{M}$ DxPCA dissolved in an aqueous solution (water $/ 5 \%$ EtOH) and loaded into CMS particles (dissolved in water $/ 5 \%$ EtOH) were applied $\left(20 \mu \mathrm{L} / \mathrm{cm}^{2}\right)$ even on porcine ear skin, followed by a storage in a skin chamber at $32{ }^{\circ} \mathrm{C}$ [35]. For investigations into deeper porcine ear skin layers, the tape stripping method was performed as previously described [36]. For these measurements, skin slices with a diameter of $4.5 \mathrm{~mm}$ were punched-out by using a punch pliers Typ3519 (R. Lühdorff GmbH Famex-Werkzeuge, Remscheid, Germany).

\subsection{EPR spectroscopy and analysis}

Cryogenic temperature $(80 \mathrm{~K})$ measurements at W-band (94 GHz) were performed on a Elexsys E680 EPR spectrometer equipped with a Teraflex EN600-1021H probe head (both Bruker Biospin, Karlsruhe, Germany). The temperature was controlled by an ITC503 (Oxford Instruments, Oxfordshire, United Kingdom). The magnetic field was calibrated by using N@C60 before each measurement [37]. Quartz capillaries with $0.87 \mathrm{~mm} / 0.7 \mathrm{~mm}$ outer/inner diameters (OD/ID) (VitroCom Inc. Mountain Lakes, NJ, USA) were used for the W-band measurements. All spectra at W-band were recorded in the 
field sweep echo (FSE) mode [38]. A $0.5 \mathrm{mT}$ pseudo modulation amplitude was used to numerically calculate first derivative spectra [39]. The characteristic longitudinal relaxation time $T_{1}$ was measured by an inversion recovery experiment [40], i.e. a inversion $\pi$-pulse followed by a Hahn echo detection sequence with incrementing time between inversion and detection.

Room temperature measurements were performed on two different X-band (9 GHz) spectrometers. For spin label mobility measurements we used a lab built spectrometer consisting of a Bruker (Rheinstetten, Germany) ER 041 MR microwave bridge controlled by a Bruker ER $048 \mathrm{R}$ microwave bridge controller, a Bruker E088 100-controlled AEG electromagnet, and a Bruker 4122 SHQE-W1 microwave resonator(Bruker Biospin, Karlsruhe, Germany). Lock-in amplification was done by a Stanford Research Systems SR810 DSP Lock-In Amplifier and the microwave frequency measured by an Agilent 53181A Frequency Counter. Here samples were placed in $2 \mathrm{~mm} / 1$ mm (OD/ID) quartz capillaries (QSIL GmbH, Langewiesen, Germany). The porcine ear skin measurements were performed after 4 and 24 hours incubation time at ambient temperature $\left(21^{\circ} \mathrm{C}\right)$ by using a Elexsys E500 spectrometer including a TMHS resonator, a tissue cell (ER 162TC-Q) and a rapid scan unit (all Bruker BioSpin, Karlsruhe, Germany). These measurements were performed in triplicate on different porcine ear skin samples. All EPR spectra were analysed with the Easyspin [41], Matlab (The MathWorks GmbH, Ismaning, Germany) toolbox. 


\section{Results and discussion}

\subsection{Micro-environment of DxPCA loaded to CMS nano-particles}

The loading into and location of DxPCA in the CMS nanoparticles were investigated by probing the DxPCA micro-environment polarity. The sensitivity of W-band EPR spectra of DxPCA on different environments is shown in Fig. 1 for the extreme cases of the highly polar solvent water and the highly apolar solvent toluene together with the spectrum of DxPCA loaded to CMS. Besides water and toluene, we have examined six additional solvents and solvent mixtures as polarity/proticity references. The $g$ - and $A$-matrices for DxPCA in the different environments are listed in table 1. We have chosen to use Reichardt's $E_{T}^{N}$ value [42] as parameter for correlating the measured magnetic parameters with the solvent polarity/proticity properites. The dependence of the $g_{x x}$ and $A_{z z}$ components on $E_{T}^{N}$ is plotted in Fig. 2. Figure 2 shows the well known trend of the $g$-matrix principal value $g_{x x}$ shifting towards lower values from apolar/aprotic to polar/protic solvents, and on other hand the nitrogen hyperfine coupling $A$-matrix principal value $A_{z z}$ shifting towards higher values [43]. The changes in $g_{x x}$ and $A_{z z}$ are governed by the proticity in polar micro-environments while in absence of hydrogen bounding the relative dielectric constant $\varepsilon_{r}$ is the main governing parameter [44]. Comparing two solvents with approximately the same $\varepsilon_{r}$, e.g. acetone and 1-propanol, the dominating influence of proticity on $g_{x x}$ and $A_{z z}$ becomes evident (see table 1).

Previously, the unmodified spin label PCA when loaded to CMS nanoparticles (PCA@CMS) was investigated [14]. There, a highly polar environment was found for PCA and it was concluded that PCA is located at the surface 
of the CMS. The magnetic parameters of DxPCA loaded to nanoparticples (DxPCA@CMS) determined here ( $g$-matrix principal values $(2.00865,2.0061$, $2.0021)$ and $A$-matrix principal values $(14,14,98) \mathrm{MHz}$ ) clearly deviate from those found for PCA@CMS in the preceding study [14]. DxPCA@CMS values represent a micro-environment of intermediate polarity as visualized in fig. 2. This corresponds to the interface region between the inner hydrophobic core and the hydrophilic outer shell of the CMS. Dx is a hydrophobic drug $(\log \mathrm{P}=1.83)$ and even though unbound PCA shows a hydrophilic behavior $(\log \mathrm{P}=-1.7)$ DxPCA $(\log \mathrm{P}=1.89)$ shows a very similar hydrophobic behavior as unmodified Dx. It is thus interesting to find DxPCA in a region of intermediate polarity within the CMS. The $g$ and hfc parameters determined above strongly depend on the relative unpaired spin density at the nitrogen and the oxygen atoms of the NO function, and, thereby, report on the micro-environment of the spin label via the electronic structure. A magnetic parameter probing complementary aspects of micro-environment properties is the the spin-lattice relaxation time $T_{1}[46,47]$. It is sensitive to molecular vibrations and, thereby, provides information on mechanical properties of the micro-environment. Thus, inversion recovery measurements were performed in addition to the EPR experiments discussed above. The corresponding time traces are shown in fig. 3. The time traces were fitted by a bi-exponential function (see Tab. 2). The obtained fit parameters are given in Tab.2. The the larger time constant $\tau_{1}$ occurring with about twice the amplitude than the shorter time constant $\tau_{2}$ was considered as the relevant $T_{1}$ time [47]. Comparison of the $T_{1}$ (i.e. $\tau_{1}$ in Tab. 2) for PCA and DxPCA in water shows very similar $T_{1}$ times for both species, which are clearly distinct 
Table 1: Magnetic Parameters of DxPCA in different solvents (Error margins in the last digit given in parenthesis), together with polarities and relative dielectric permetivities of the used solvents; (p) protic, (a) is aprotic.

\begin{tabular}{|c|c|c|c|c|}
\hline Solvent & $g$-matrix & $A$-matrix $(\mathrm{MHz})$ & $E_{T}^{N}[42]$ & $\varepsilon_{r}[45]$ \\
\hline & $g_{x x}, g_{y y}, g_{z z}$ & $A_{x x}, A_{y y}, A_{z z}$ & & \\
\hline CMS & $2.00865(2), 2.0061(2), 2.0021(4)$ & $14,14,98(1)$ & & \\
\hline Water (p) & $2.00810(2), 2.0060(2), 2.0021(4)$ & $15,15,104(1)$ & 1 & 80.4 \\
\hline Ethanol(p) & $2.00844(4), 2.0060(2), 2.0021(5)$ & $15,15,101(1)$ & 0.654 & 24.3 \\
\hline 1-propanol(p) & $2.00853(3), 2.0060(5), 2.0021(1)$ & $13,13,101(1)$ & 0.617 & 20.1 \\
\hline 1-decanol(p) & $2.00860(2), 2.0061(3), 2.0021(3)$ & $13,12,99(1)$ & 0.519 & 8.1 \\
\hline $\operatorname{DMSO}(\mathrm{a})$ & $2.00864(4), 2.0060(3), 2.0021(1)$ & $15,15,100(1)$ & 0.444 & 46.68 \\
\hline Acetone(a) & $2.00870(3), 2.0061(2), 2.0022(1)$ & $1515,96(1)$ & 0.355 & 20.7 \\
\hline Methyl formate(a) & $2.00865(5), 2.0060(2), 2.0021(1)$ & $15,15,98(1)$ & 0.346 & 8.5 \\
\hline Toluene(a) & $2.00900(5), 2.0061(3), 2.0021(3)$ & $15,15,95(1)$ & 0.099 & 2.4 \\
\hline PCA@CMS [14] & $2.00890,2.00600,2.0012$ & $30,35,72$ & & \\
\hline PCA@Water (p) & $2.00805(3), 2.00596(2), 2.00212(2)$ & $15,15,105(1)$ & 1 & 80.4 \\
\hline
\end{tabular}

from the $T_{1}$ time for DxPCA@CMS. This corroborates the finding above that DxPCA experiences an environment different from the water phase and is within the CMS.

\subsection{Spin probe mobility in CMS nanoparticles}

The spectral shape arising for room temperature measurements, as for the low temperature, again depends on the $g$ - and $A$-matrix as well as the experimental microwave frequency, but in addition is strongly determined by 
Table 2: Spin lattice relaxation time $T_{1}$ obtained by bi-exponential fitting of inversion recovery time traces to the function $f(t)=1-2\left(A_{1} e^{\left(-\tau / \tau_{1}\right)}+A_{2} e^{\left(-\tau / \tau_{2}\right)}\right)$.

\begin{tabular}{lcccc}
\hline Material & $A_{1}$ & $\tau_{1}$ (us) & $A_{2}$ & $\tau_{2}$ (us) \\
DxPCA@CMS & 0.67 & 240 & 0.33 & 31 \\
DxPCA & 0.70 & 379 & 0.30 & 26 \\
PCA & 0.68 & 398 & 0.32 & 32 \\
\hline
\end{tabular}

the mobility of the spin probe $[48,49]$. Variations in the mobility due to the different spin probe micro-environments manifest clearly in the line-width, line shape and hyperfine splitting in X-band $(9 \mathrm{GHz})$ spectra recorded at ambient temperatures. The spectrum of small free nitroxide spin probes in isotropic solution with low viscosity, corresponding to rotational correlation times $\left(\tau_{\text {corr }}\right)$ of 100 ps or faster at X-band frequencies, consists of three sharp lines. For slower tumbling due to a more viscous or anisotropic environment, the three lines become broader. The characteristic rotational correlation time $\tau_{\text {corr }}$ for this regime is in the range of $300-1000$ ps at X-band frequencies. The spectra become significantly broadened for further immobilized spin probes, where particularly the low field peak ( $1^{\text {st }}$ line) and high field peak ( $3^{\text {rd }}$ line) are sensitive for broadening. Quantitative values for $\tau_{\text {corr }}$ can be extracted from the spectra by simulation [48]. The optimum EPR frequency band for monitoring spin label dynamics depends on the relevant rotational correlation times. Here, experiments at X-band frequencies were found to be adequate. Both the spectra of PCA and DxPCA show three sharp lines in aqueous solution with approximately equal height (Figure: 4, top and middle spectra) in agreement with the small effective radius and the low viscosity solvent. 
The slightly larger effective radius of DxPCA compared to PCA results in a slower rotation and in consequence yields the slightly broadened and less intense high field peak for DxPCA. Spectral simulation reveals rotational correlation times of about 10 ps and 80 ps for PCA and DxPCA, respectively. These numbers are in good agreement with the rotational correlation times calculated for both molecules using the Stokes-Einstein equation $\left(\tau=\frac{8 \pi \eta R^{3}}{6 k_{T} T}\right)$. In the DxPCA spectrum a further spectral contribution can be seen as a broad unstructured line between the first and the second sharp line. This contribution is due to the low solubility of Dx in water, causing a partial aggregation of DxPCA. These aggregates likely contain a high DxPCA concentration with distances between DxPCA molecules giving rise to substantial spin-spin coupling, which in turn results in a strongly broadened spectrum devoid of the otherwise characteristic hyperfine sturcture.

The spectrum of DxPCA@CMS (Figure: 4, bottom spectrum) clearly consists of two components. The first component consists of the characteristic three narrow and sharp lines. The second component is in this case a broad signal well visible to the left of the first sharp line. Again, the sharp lines represent a highly mobile fraction of DxPCA, indicative for the presence of free DxPCA in solution not loaded to CMS. The broad spectral contribution (see fig. 5, bottom spectrum) was extracted by subtracting the free DxPCA component from the DxPCA@CMS spectrum. The resulting spectral component is representative for the intermediate mobility range between the fast tumbling and the solid state regime and can be qualitatively simulated under the assumption of a rotational correlation time $\tau_{\text {corr }} \approx 7$ ns. This number clearly shows a severely slowed rotational motion of DxPCA loaded 
to CMS compared to DxPCA in aqueous solution. An interpretation of the rotational correlation time in terms of the DxPCA mobility within CMS is possible, when first considering the rotational correlation time of the CMS nanoparticles. Their average diameter was determined by dynamic light scattering $(\mathrm{DLS})$ as $r_{C M S} \simeq 18 \mathrm{~nm}$. Again using the Stokes-Einstein equation $\left(\tau=\frac{8 \pi \eta R^{3}}{6 k_{T} T}\right)$, we calculate a rotational correlation time $\left(\tau_{C M S} \simeq 1 \mu \mathrm{s}\right)$. Such a very slow rotational correlation time yields in simulations spectra virtually indistinguishable from the solid state limit. Thus, we can conclude that the rotational motion of the whole CMS nanoparticles has no influence on the observed spectral shape and the $\tau_{\text {corr }} \approx 7 \mathrm{~ns}$ derived for DxPCA@CMS exclusively reports the residual mobility of DxPCA within the CMS. The deconvolution of the DxPCA@CMS spectrum allows a further conclusion on the relative amounts of free DxPCA in solution and DxPCA loaded to CMS. Even though the peak amplitudes of the narrow three lines for the free DxPCA component are substantially larger than that of the broad DxPCA@CMS component, the ratio between free and loaded DxPCA amounts to 1:20. This shows that the equilibrium between DxPCA@CMS and free DxPCA in the aqueous solution is strongly shifted to DxPCA loaded to the CMS.

\subsection{Skin penetration of DxPCA loaded to $C M S$}

The penetration of DxPCA@CMS into porcine ear skin was investigated again by room temperature X-band EPR in comparison to DxPCA dissolved in a water $/ 5 \%$ EtOH solution 4 and 24 hours after topical application of both sample types. In both cases an increase of the EPR signal can be seen between the measurements performed after $4 \mathrm{~h}$ incubation and that after 
$24 \mathrm{~h}$ (fig. 6). For DxPCA dissolved in a water $/ 5 \%$ EtOH solution one single broadened peak is hardly visible in the spectrum recorded after $4 \mathrm{~h}$ and becomes better visible after $24 \mathrm{~h}$ (fig. 6 left) at the spectral position corresponding to the center line of the typical three line nitroxide spectrum. In contrast to this result, the spectra for DxPCA@CMS show for both times the characteristic three line nitroxide EPR spectrum (fig. 6 right). The intensity of this spectral contribution remains largely unchanged. In addition to the narrow line spectrum, a second broadened contribution is visible at both times, mainly as intensity between the first and second peaks of the narrow line spectrum. This spectral contribution increases in intensity relative to the narrow component in the spectrum taken after $24 \mathrm{~h}$. We assign the two spectral contributions visible for DxPCA@CMS again as before. The three line spectrum represents free DxPCA while the broadened component is due to DxPCA still incorporated into CMS. The increased intensity of the broad component after $24 \mathrm{~h}$ compared to the $4 \mathrm{~h}$ spectrum recorded for DxPCA@CMS incubation on skin reports thus an enhanced CMS nanoparticle penetration into the porcine ear skin with time.

Whether DxPCA@CMS and the free DxPCA penetrate into the porcine ear skin or only form a surface layer can be tested by the tape stripping method (see e.g. [36]). Figure 7 shows X-band EPR spectra of DxPCA applied onto porcine ear skin after $24 \mathrm{~h}$ incubation time and removal of the supernatant followed by removal of 1,2 , and 3 tapes. After removal of the first layers of the SC a strong difference in the penetration efficiency for two different DxPCA application schemes becomes apparent: DxPCA dissolved in the water $/ 5 \%$ EtOH solution solution shows for the whole porcine ear 
skin one broadend EPR signal, which strongly diminishes after removing the first adhesive tape and has virtually disappeared after removing the second. For DxPCA@CMS the EPR signal decreases gradually after stripping of one to three adhesive tapes. Within the limited signal-to-noise ratio, the ratio between the free DxPCA and the DxPCA@CMS components seems to be unchanged (Fig. 7). These results are in agreement with the investigation of Küchler et al. showing the skin penetration of CMS using a lipophilic fluorescent dye [18] .

A remaining question is whether the CMS particles transport only DxPCA loaded into them or whether they act as a general penetration enhancer promoting co-transport of the DxPCA outside them. To address this question we prepared a mixture of $90 \mu \mathrm{M}$ DxPCA aqueous/5\% EtOH solution and empty CMS particles. This mixture was applied in the same way as the DxPCA@CMS sample onto porcine ear skin, incubated for 24 hours and subjected to tape stripping (fig. 7C). Again only a single line EPR signal is visible for the porcine ear skin before and after tape stripping, and signal intensity is - if at all - only weakly increased compared to the aqueous DxPCA solution without CMS (fig. 7A). Thus, a co-transport of DxPCA by CMS can be only of minor importance and the deeper penetration of DxPCA into the skin when applied after loading into CMS is in the vast majority due to direct transport as cargo within the CMS.

\section{Conclusion}

The presented investigations have shown that EPR is able to provide evidence for the loading of Dx into CMS nanoparticles and for the Dx loca- 
tion within the nanoparticles. Thereby, we have made use of complementary magnetic parameters of the spin label PCA covalently attached to the drug Dexamethasone. The $g$ - and $A$-matrices of DxPCA report on the polarity/proticity of its micro-environment while the spin-lattice relaxation time $T_{1}$ probes vibrational properties of the DxPCA micro-environment. These magnetic parameters were measured by pulsed high-field EPR and clearly show a location of DxPCA within the CMS nanoparticles in a region of intermediated polarity, likely the interface between the hyrdophobic and hydrophilic shells of the CMS. Furthermore, we determined dynamical information on the drug in aqueous solution and loaded into the CMS nanocarriers using continuous wave EPR at ambient temperature. The differences found in the dynamics between freely dissolved DxPCA and DxPCA loaded into CMS was used to analyse the drug/carrier penetration behaviour into skin. Thereby, we could show that the penetration of the drug into the porcine ear skin is facilitated only for the drug loaded into the CMS and that a cotransport of the drug through a lowered skin barrier by CMS is of only minor importance if at all relevant.

\section{Chemical compounds studied in this article}

- Dexamethasone (PubChem CID: 5743)

- 3-(Carboxy)-2,2,5,5-tetramethyl-1-pyrrolidinyloxy (PubChem CID: 519874)

- Ethanol (PubChem CID: 702)

- 1-propanol (PubChem CID: 1031)

- 1-decanol (PubChem CID: 8174) 
- DMSO (PubChem CID: 679)

- Acetone (PubChem CID: 180)

- Methyl formate (PubChem CID: 7865)

- Toluene (PubChem CID: 1140)

\section{Acknowledgments}

The authors acknowledge support from Deutsche Forschungsgemeinschaft (DFG) via SFB 1112, Project B01.

\section{References}

[1] M. Schafer-Korting, W. Mehnert, H. Korting, Lipid nanoparticles for improved topical application of drugs for skin diseases, Adv. Drug Deliv. Rev. 59 (2007) 427-443.

[2] A. Wilczewska, K. Niemirowicz, K. Markiewicz, H. Car, Nanoparticles as drug delivery systems, Pharmacol. Rep. 64 (2012) 1020-1037.

[3] S. Gupta, R. Bansal, N. S. Gupta, Jindal, A. Jindal, Nanocarriers and nanoparticles for skin care and dermatological treatments, Indian Dermatol Online J. 4 (2013) 267-272.

[4] J. Lademann, N. Otberg, H. Richter, U. Jacobi, H. Schaefer, U. BlumePeytavi, W. Sterry, Follicular penetration. An important pathway for topically applied substances, Hautarzt 54 (2003) 321-323. 
[5] J. Lademann, H. Richter, A. Teichmann, N. Otberg, U. Blume-Peytavi, J. Luengo, B. Weiß, U. F. Schaefer, C. M. Lehr, R. Wepf, W. Sterry, Nanoparticles - An efficient carrier for drug delivery into the hair follicles, European Journal of Pharmaceutics and Biopharmaceutics 66 (2007) $159-164$.

[6] A. Patzelt, H. Richter, F. Knorr, U. Schafer, C. Lehr, L. Dahne, W. Sterry, J. Lademann, Selective follicular targeting by modification of the particle sizes, J. Control Release, 150 (2011) 45-48.

[7] A. Vogt, U. Blume-Peytavi, Selective hair therapy: Bringing science to the fiction, Exp. Dermatol, 23 (2014) 83-86.

[8] W. D. Jong, P. Borm, Drug delivery and nanoparticles: Applications and hazards, Int. J. Nanomedicine 3 (2008) 133-149.

[9] M. R. Radowski, A. Shukla, H. von Berlepsch, C. Böttcher, G. Pickaert, H. Rehage, R. Haag, Supramolecular aggregates of dendritic multishell architectures as universal nanocarriers, Angewandte Chemie International Edition 46 (2007) 1265-1269.

[10] J. F. Coelho, P. C. Ferreira, P. Alves, R. Cordeiro, A. C. Fonseca, J. R. Góis, M. H. Gil, Drug delivery systems: Advanced technologies potentially applicable in personalized treatments, EPMA Journal 1 (2010) 164-209.

[11] W. B. Liechty, D. R. Kryscio, B. V. Slaughter, N. A. Peppas, Polymers for drug delivery systems, Annu. Rev. Chem. Biomol. Eng. 1 (2010) $149-173$. 
[12] Zhang, Ying, Chan, HF, K. Leong, Advanced materials and processing for drug delivery: The past and the future, Advanced Drug Delivery Reviews 65 (2013) $104-120$.

[13] D. Kapoor, A. Bhatia, R. Kaur, R. Sharma, G. Kaur, S. Dhawan, PLGA: A unique polymer for drug delivery, Ther. Deliv. 6 (2016) 41-58.

[14] S. Haag, E. Fleige, M. Chen, A. Fahr, C. Teutloff, R. Bittl, J. Lademann, M. Schafer-Korting, R. Haag, M. Meinke, Skin penetration enhancement of core-multishell nanotransporters and invasomes measured by electron paramagnetic resonance spectroscopy, International Journal of Pharmaceutics 416 (2011) $223-228$.

[15] R. Haag, Supramolecular drug-delivery systems based on polymeric coreshell architectures, Angew. Chem. Int. Ed. Engl. 43 (2004) 278-282.

[16] A. Boreham, M. Pfaff, E. Fleige, R. Haag, U. Alexiev, Nanodynamics of dendritic core-multishell nanocarriers, Langmuir 30 (2014) 1686-1695.

[17] S. Lohan, N. Icken, C. Teutloff, S. Saeidpour, R. Bittl, J. Lademann, E. Fleige, R. Haag, S. Haag, M. Meinke, Investigation of cutaneous penetration properties of stearic acid loaded to dendritic core-multi-shell (CMS) nanocarriers, International Journal of Pharmaceutics 501 (2016) $271-277$.

[18] S. Küchler, M. R. Radowski, T. Blaschke, M. Dathe, J. Plendl, R. Haag, M. Schäfer-Korting, K. D. Kramer, Nanoparticles for skin penetration enhancement - a comparison of a dendritic core-multishell- 
nanotransporter and solid lipid nanoparticles, Eur. J. Pharm. Biopharm, 71 (2009) 243-50.

[19] K. A. Earle, J. K. Moscicki, M. Ge, D. E. Budil, J. H. Freed, 250-GHz electron spin resonance studies of polarity gradients along the aliphatic chains in phospholipid membranes, Biophysical journal 66 (1994) 12131221.

[20] H.-J. Steinhoff, M. Pfeiffer, T. Rink, O. Burlon, M. Kurz, J. Riesle, E. Heuberger, K. Gerwert, D. Oesterhelt, Azide reduces the hydrophobic barrier of the bacteriorhodopsin proton channel, Biophysical Journal 76 (1999) $2702-2710$.

[21] S. Haag, M. Chen, D. Peters, C. Keck, B. Taskoparan, A. Fahr, C. Teutloff, R. Bittl, J. Lademann, M. Schafer-Korting, M. Meinke, Nanostructured lipid carriers as nitroxide depot system measured by electron paramagnetic resonance spectroscopy, International Journal of Pharmaceutics 421 (2011) 364-369.

[22] U. Bhardwaj, D. J. Burgess, Physicochemical properties of extruded and non-extruded liposomes containing the hydrophobic drug dexamethasone, International Journal of Pharmaceutics 388 (2010) 181 - 189.

[23] C. Altenbach, S. L. Flitsch, H. G. Khorana, W. L. Hubbell, Structural studies on transmembrane proteins. 2. spin labeling of bacteriorhodopsin mutants at unique cysteines, Biochemistry 28 (1989) 7806-7812.

[24] M. Unbehauen, K. Walker, S. Lohan, M. Meinke, R. Zimmer, H. U. Reißig, R. Haag, Spin-labelling of dexamethasone for electron- 
paramagnetic resonance (EPR) spectroscopy on hacat cells, In preparation.

[25] S. Saeidpour, S. B. Lohan, A. Solik, V. Paul, R. Bodmeier, G. Zoubari, R. Bittl, M. C. Meinke, C. Teutloff, Drug distribution in nanostructured lipid particles, Submitted to European Journal of Pharmaceutics and Biopharmaceutics.

[26] T. Kamawamura, S. Matsunami, T. Yonezawa, Solvent effects on the g-value of di-t-butyl nitric oxide, Bulletin of the Chemical Society of Japan 40 (1967) 1111-1115.

[27] O. H. Griffith, P. J. Dehlinger, S. P. Van, Shape of the hydrophobic barrier of phospholipid bilayers (evidence for water penetration in biological membranes), The Journal of Membrane Biology 15 (1974) 159-192.

[28] S. Kempe, H. Metz, K. Mäder, Application of electron paramagnetic resonance (EPR) spectroscopy and imaging in drug delivery research chances and challenges, European Journal of Pharmaceutics and Biopharmaceutics 74 (2010) $55-66$.

[29] G. Martini, L. Ciani, Electron spin resonance spectroscopy in drug delivery, Phys. Chem. Chem. Phys. 11 (2009) 211-254.

[30] T. Herrling, J. Fuchs, J. Rehberg, N. Groth, UV-induced free radicals in the skin detected by ESR spectroscopy and imaging using nitroxides, Free Radical Biology and Medicine 35 (2003) 59- 67.

[31] S. Haag, M. Chen, B. Taskoparan, R. Bittl, C. Teutloff, R. Wenzel, A. Fahr, J. Lademann, M. Schäfer-Korting, M. Meinke, Stabilization 
of reactive nitroxides using invasomes to allow prolonged electron paramagnetic resonance measurements skin, Pharmacol. Physiol. 24 (2011) 312-321.

[32] S. H, Yalkowsky, Y. He, P. Jain, Handbook of Aqueous Solubility Data, Second Edition, CRC, 2010.

[33] W. Meyer, R. Schwarz, K. Neurand, The skin of domestic mammals as a model for the human skin, with special reference to the domestic pig, Curr Probl Dermatol 7 (1978) 39-52.

[34] S. Haag, A. Bechtel, M. Darvin, F. Klein, N. Groth, M. Schafer-Korting, R. Bittl, J. Lademann, W. Sterry, M. Meinke, Comparative study of carotenoids, catalase and radical formation in human and animal skin, Skin Pharmacol. Physiol. 23 (2010) 306-312.

[35] A. Vogt, F. Rancan, S. Ahlberg, B. Nazemi, C. Choe, M. Darvin, et al., Interaction of dermatologically relevant nanoparticles with skin cells and skin, Beilstein. J. Nanotechnol. 5 (2014) 2363-2373.

[36] J. Lademann, M. Meinke, S. Schanzer, H. Richter, M. Darvin, S. Haag, J. Fluhr, H. Weigmann, W. Sterry, A. Patzelt, In vivo methods for the analysis of the penetration of topically applied substances in and through the skin barrier, Int. J. Cosmet. Sci. 34 (2012) 551-559.

[37] A. Weidinger, M. Waiblinger, B. Pietzak, T. A. Murphy, Atomic nitrogen in C60:N@C60, Applied Physics A 66 (1998) 287-292.

[38] E. L. Hahn, Spin echoes, Phys. Rev. 80 (1950) 580-594. 
[39] J. S. Hyde, M. Pasenkiewicz-Gierula, A. Jesmanowicz, W. E. Antholine, Pseudo field modulation in EPR spectroscopy, Applied Magnetic Resonance 1 (1990) 483-496.

[40] E. Hoffmann, A. Schweiger, Inversion-recovery detected EPR, Applied Magnetic Resonance 9 (1995) 1-22.

[41] S. Stoll, A. Schweiger, Easyspin, a comprehensive software package for spectral simulation and analysis in EPR, Journal of Magnetic Resonance $178(2006) 42-55$.

[42] Reichardt, Christian, Welton, Thomas, Empirical Parameters of Solvent Polarity, Wiley-VCH, 2010, pp. 425-508.

[43] M. Plato, H. J. Rteinhoff, C. Wegener, J. T. Törring, A. Savitsky, K. Möbius, Molecular orbital study of polarity and hydrogen bonding effects on the g and hyperfine tensors of site directed no spin labelled bacteriorhodopsin, Molecular Physics 100 (2014) 3711-3721.

[44] D. Marsh, Spin-label EPR for determining polarity and proticity in biomolecular assemblies: Transmembrane profiles, Applied Magnetic Resonance 37 (2010) 435-454.

[45] R. Owenius, M. Engström, M. Lindgren, M. Huber, Influence of solvent polarity and hydrogen bonding on the EPR parameters of a nitroxide spin label studied by $9-\mathrm{GHz}$ and $95-\mathrm{GHz}$ EPR spectroscopy and DFT calculations, The Journal of Physical Chemistry A 105 (2001) 1096710977. 
481

[46] J. Du, G. Eaton, S. Eaton, Temperature, orientation, and solvent dependence of electron spin-lattice relaxation rates for nitroxyl radicals in glassy solvents and doped solids, Journal of Magnetic Resonance, Series A 115 (1995) $213-221$.

[47] S. S. Eaton, G. R. Eaton, Distance Measurements in Biological Systems by EPR, Kluwer Academic Publishers, 2002.

[48] L. J. Berliner (Ed.), Spin Labeling (Theory and Application), Academic Press, 1976.

[49] L. J. Berliner (Ed.), Spin Labeling II (Theory and Application), Academic Press, 1979. 


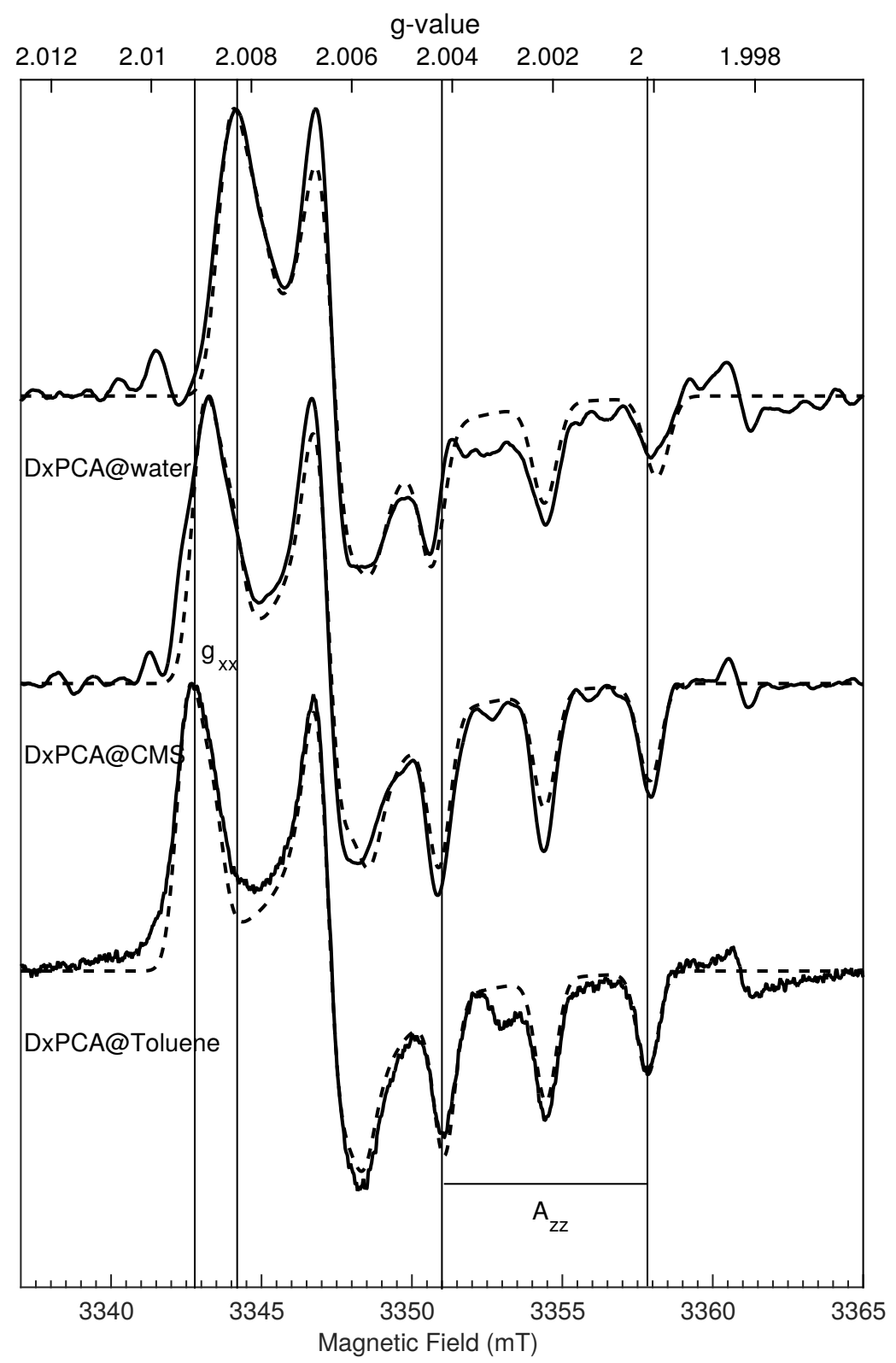

Figure 1: W-Band EPR spectra of DxPCA in different environments at $80 \mathrm{~K}$; solid line: experiment, dashed line: simulation, All spectra were normalised to a frequency of $94 \mathrm{GHz}$ 


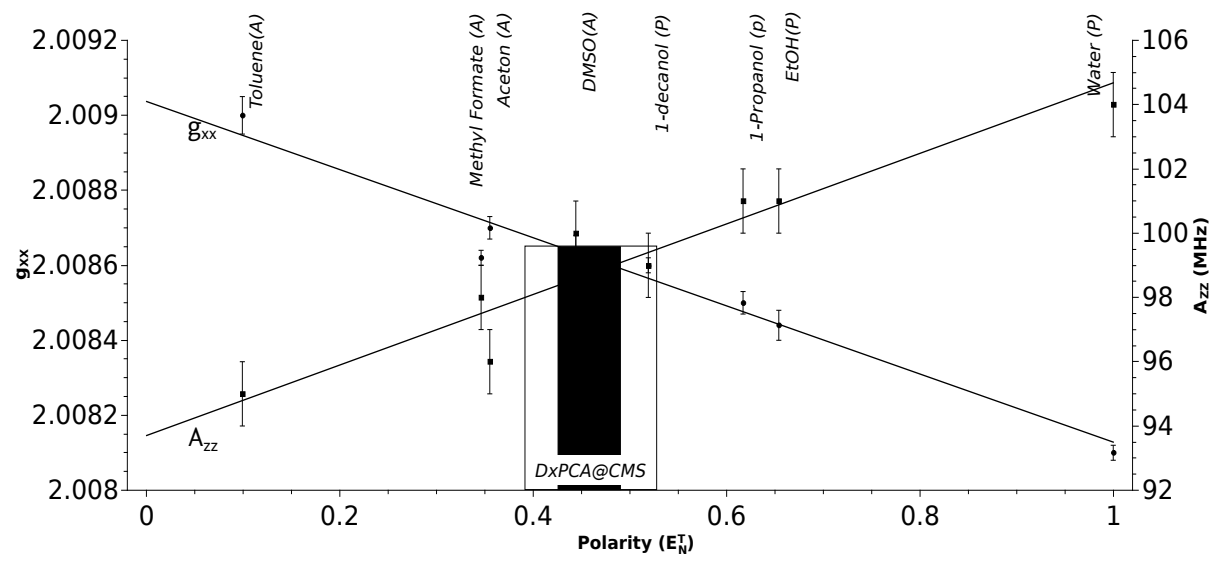

Figure 2: Dependence of $g_{x x}$ and $A_{z z}$ of DxPCA on different solvents and linear fits (both lines, respectively). The areas filled in black and white mark the polarity range compatible with the DxPCA@CMS $g_{x x}$ and $A_{z z}$ parametrers, repectively 


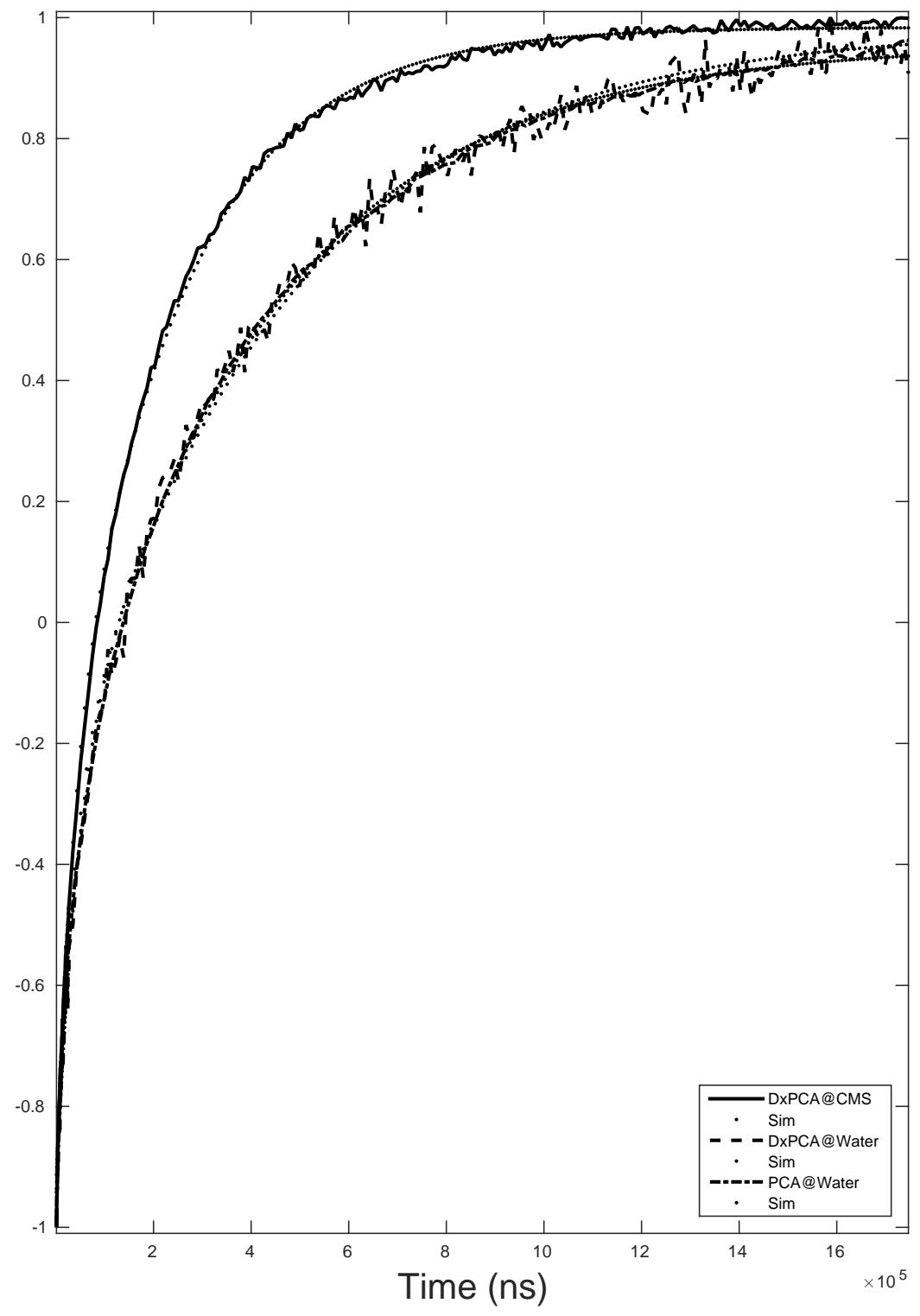

Figure 3: Inversion recovery spin-lattice relaxation time $\left(T_{1}\right)$ measurement for DxPCA@CMS (solid line), DxPCA@Water(dash line) and PCA@Water(dot dash) at W-band, $80 \mathrm{~K}$; solid line: experiment, dashed line: bi-exponential fit) 


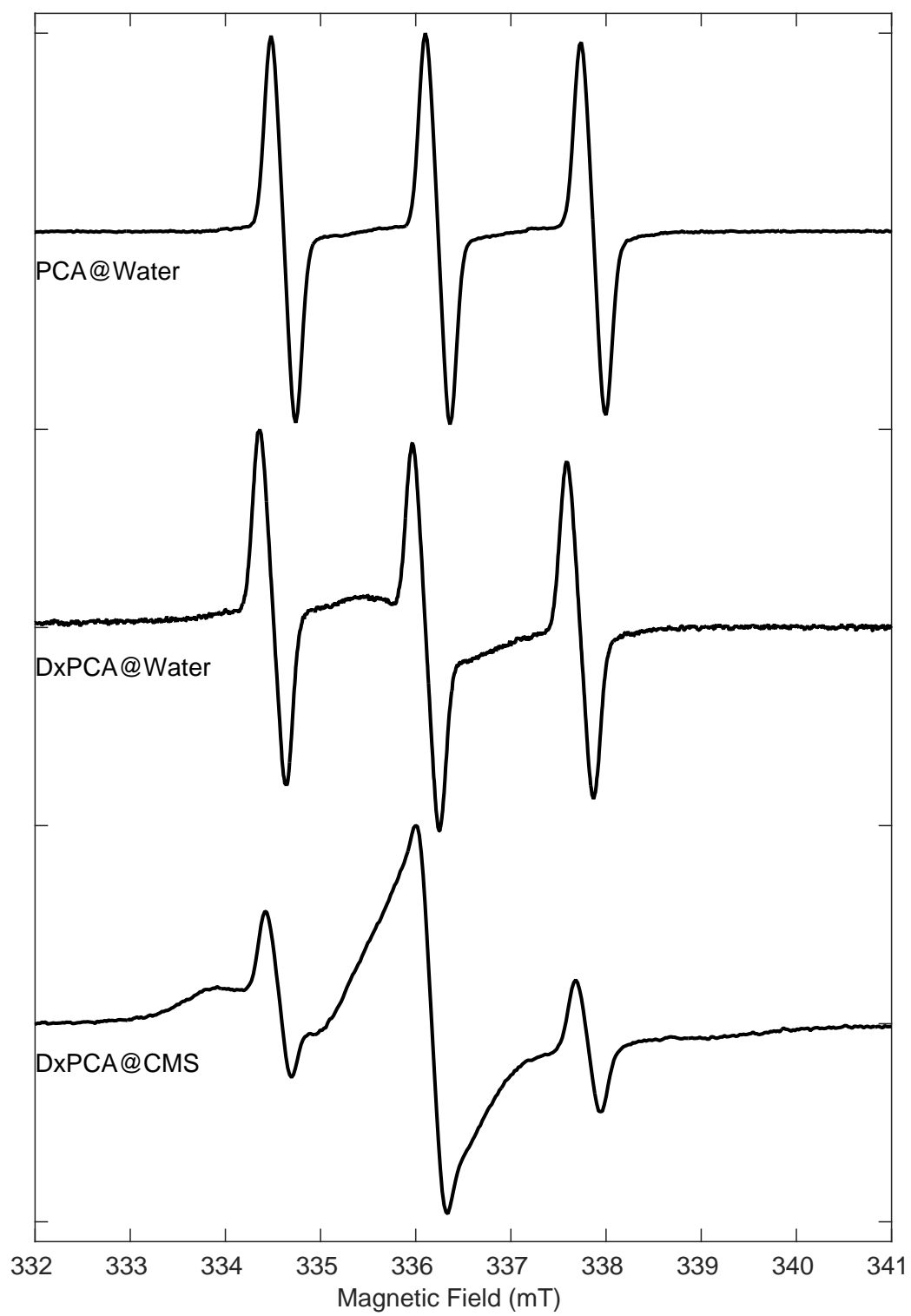

Figure 4: EPR spectra DxPCA@CMS (bottom spectrum), DxPCA in water (middle spectrum) and PCA in water (top spectrum) measured at X-band and room temperature. Experimental parameters are for DxPCA@CMS: microwave power: $50 \mathrm{mw}$, field modulation amplitude: $3 \mathrm{G}$; 8 for DxPCA in water: microwave power: $20 \mathrm{~mW}$, field modulation amplitude: $5 \mathrm{G}$, PCA in water: microwave power: $25 \mathrm{~mW}$, field modulation amplitude: $3 \mathrm{G}$ ). All spectra were normalised to a frequency of $9.4 \mathrm{GHz}$ 


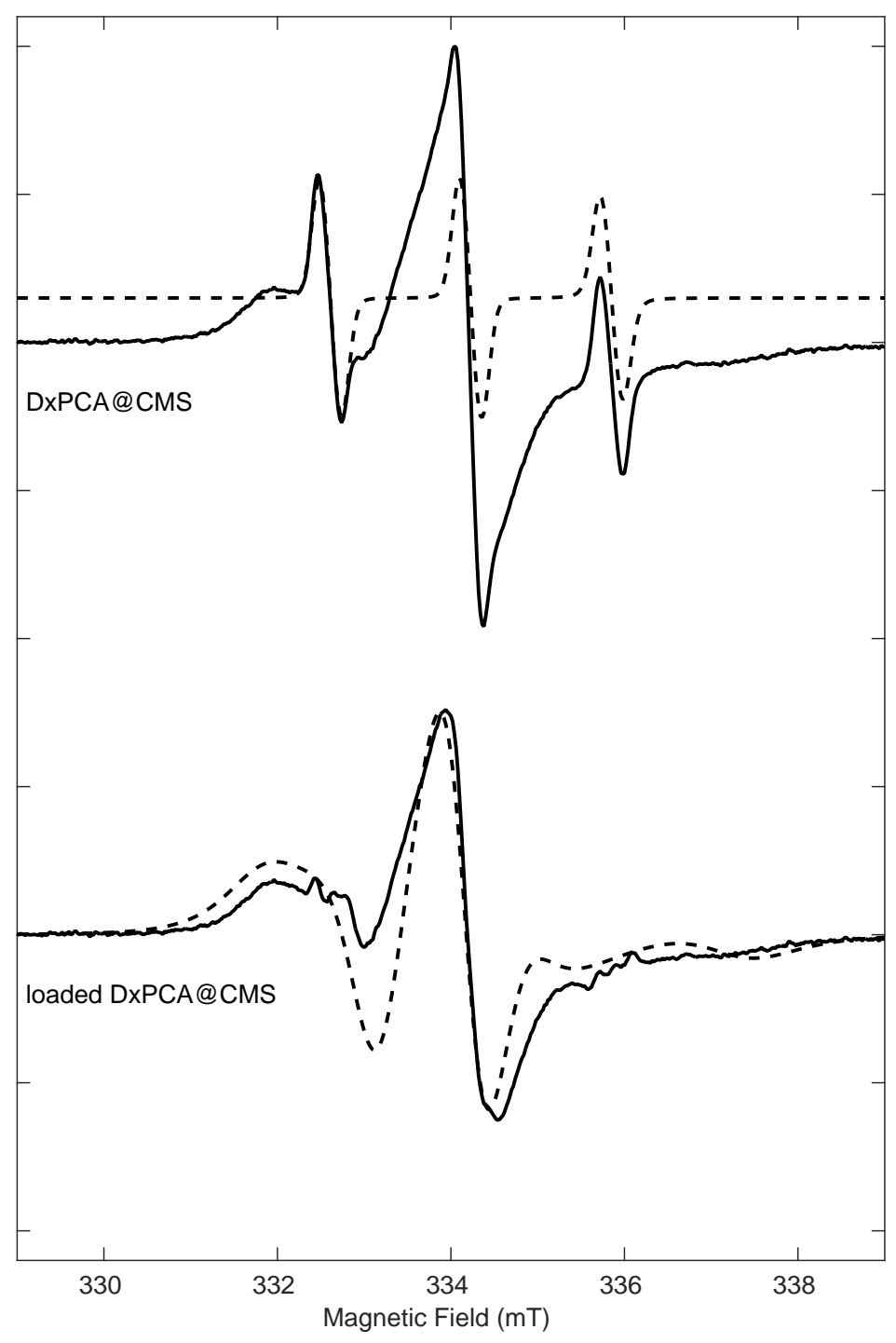

Figure 5: Deconvolution of the DxPCA@CMS spectrum into the free DxPCA spectral contribution and a partly immobilised DxPCA spectral contribution. The experimental DxPCA@CMS spectrum (top spectra) is as in fig. 4; the free DxPCA in water spectrum (top dash line) is a simulation of corresponding spectrum in fig. 4. The resulting spectrum after subtractign is given by the bottom line. 

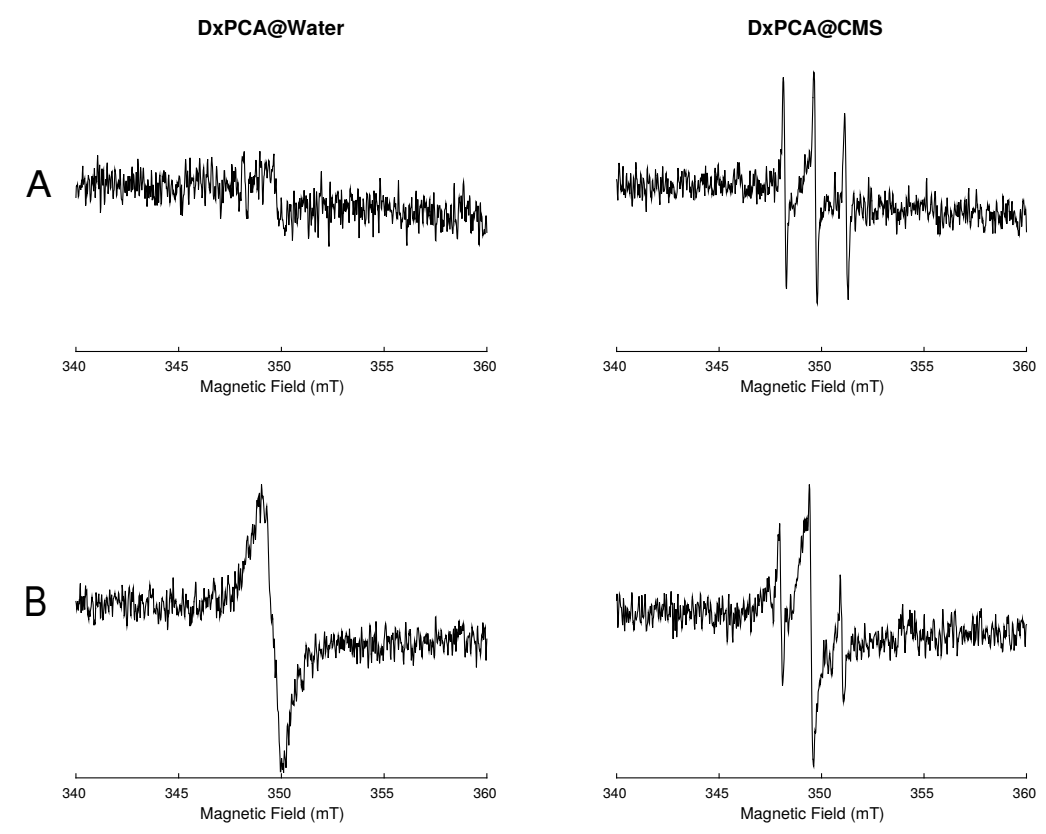

Figure 6: EPR spectra of DxPCA after topical application on porcine ear skin: (left) DxPCA dissolved in a water $/ 5 \%$ EtOH solution $(90 \mu \mathrm{M})$ and (right) DxPCA@CMS, both after an incubation time of $4 \mathrm{~h}(\mathrm{~A})$ and $24 \mathrm{~h}(\mathrm{~B})$. Each spectrum is an average of three measurements at X-band and room temperature. 


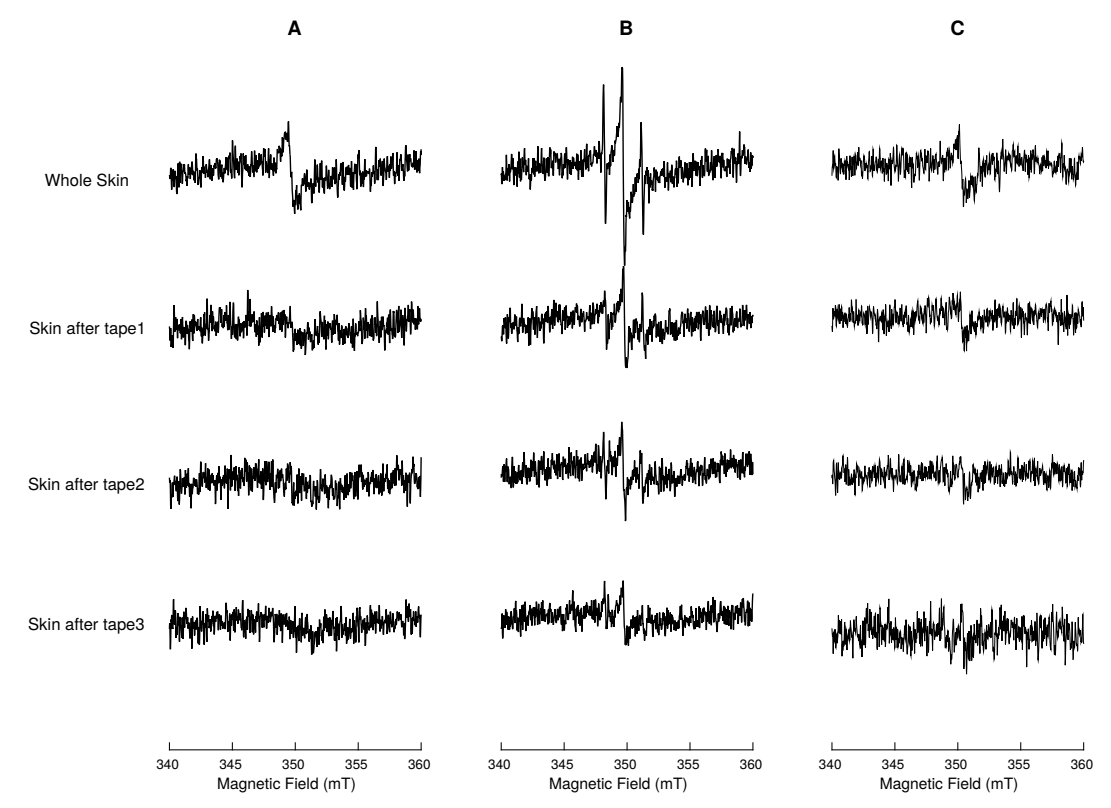

Figure 7: EPR spectra of DxPCA after topical application on porcine ear skin (A): DxPCA dissolved in a water $/ 5 \%$ EtOH solution $(90 \mu \mathrm{M}),(\mathrm{B})$ DxPCA@CMS, and $(\mathrm{C})$ a mixture of DxPCA dissolved in a water $/ 5 \%$ EtOH solution $(90 \mu \mathrm{M})$ and empty CMS particles, all after an incubation time of $24 \mathrm{~h}$ and after the indicated numbers of tape stripping. Each spectrum is an average of three measurements at $\mathrm{X}$-band and room temperature. 